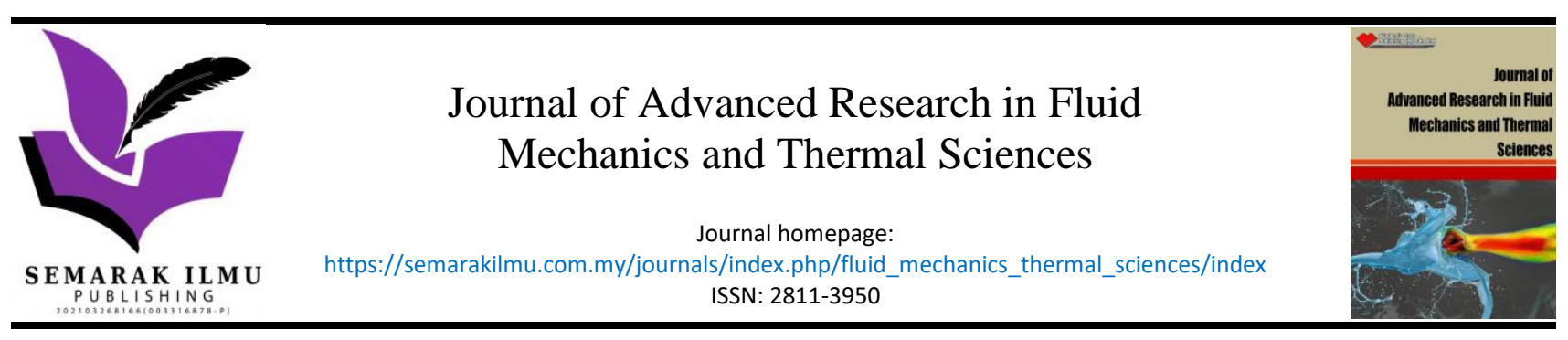

\title{
A Numerical Simulation Under Milk Fouling in A Plate Heat Exchanger in The Presence of a Porous Medium
}

\author{
Mohamed Sannad ${ }^{1,}{ }^{*}$, Youcef Mehdi ${ }^{2,3}$, Afaf Zaza ${ }^{1,4}$, Youness El Hammami ${ }^{1}$, Youssef Idihya ${ }^{1}$, \\ Othmane Benkortbi ${ }^{5}$ \\ 1 Laboratory of Mechanics, Processes, Energy and Environment, National School of Applied Sciences, Ibn Zohr University, Agadir, BP 1136, \\ Morocco \\ 2 University of Science and Technology Houari Boumediene (USTHB), Faculty of Mechanical and Process Engineering (FGMGP), Laboratory of \\ Transfer Phenomena, B.P. 32, El-Alia, Bab-Ezzouar 16000, Algiers, Algeria \\ 3 University of Médéa, Faculty of Sciences, Department of Matter Sciences, Ain D’Heb Médéa 26000, Algeria \\ 4 Department of Thermal Systems, IRESEN- Green Energy Park, Km 2 Route Régionale R206, Benguerir, Morocco \\ 5 University of Médéa, Faculty of Technology, Department of Process and Environmental Engineering, Ain D’Heb Médéa 26000, Algeria
}

\section{ARTICLE INFO}

\section{Article history:}

Received 22 July 2021

Received in revised form 5 December 2021

Accepted 9 December 2021

Available online 15 January 2021

\section{Keywords:}

PHE; milk heat treatment; fouling model; dynamic and thermal aspects; fluent code; CFD

\section{ABSTRACT}

Fouling is a known dairy industry issue during thermal treatments using a plate heat exchanger (PHE) it directly affects not only energy costs and maintenance but also production, energy and water losses during cleaning operations. Therefore, a considerable attention has been paid to the modelling of the fouling phenomena in order to improve the PHE performances. In this paper, a mathematical model describing the steady state was established for the flow at the macroscopic scale; the model account for constant and turbulent flows between two flat parallel plates at imposed temperature in the presence of a porous medium. The closure $k-\varepsilon$ model has been chosen and the conservation of equations has been developed. The numerical procedure adopted was based on the finite volume method on the Fluent CFD software. The accuracy of the Fluent software was validated by comparing our results with those previously published in the literature. The numerical results showed a good reliability of the developed code for both laminar and turbulent regimes of the studied phenomenon. It was able to capture the dynamic and the thermal aspects of the process. The maximum velocity at the output of the channel increased when the thickness of the porous medium was increased. An increase of the average temperature over the whole length of the channel was observed; while a decrease of the average temperature at the outlet of the channel was noticed. The deposit fouling caused additional thermal resistance. Finally, a decrease of the temperature gradient and Nusselt number in the medium of the channel was observed.

\section{Introduction}

Food products undergoing transformations was increasingly developed to display both an organoleptic quality and a desirable texture. The equipment manufacturers as well as the

\footnotetext{
* Corresponding author.

E-mail address: mohamedsannad@gmail.com
}

https://doi.org/10.37934/arfmts.91.1.117 
transformer attempt to define the process lines providing the desired product in optimal health and economic conditions taking into account the fouling phenomena. Owing to the diversity, the complexity and the economic problems it caused in petrochemical and food industries fouling has attracted the interest of many researchers. It is defined as the accumulation of materials and undesirable substances over a heated surface and is considered one of the major unsolved problems in the field of heat exchangers, (HE) $[1,2]$.

In the industrial companies, the $\mathrm{HE}$ is an essential element of any energy control policy. A big part of the heat energy $90 \%$ used in industrial processes transit at least once by HE. They are used in various industrial sectors (chemistry, food, etc.).

A considerable effort has been supplied to analyze and understand the fouling behavior. During the operation, the HE became fouled with a buildup of deposits of one or several components of milk on the HE transfers surfaces. As a result, the thermal resistance increased lowering the performances of the HE. The fouling layer blocked the passage of the fluid thus reducing the heat transfer rate below the desired heat treatment [1].

Milk fouling which is of insulating nature and very complex is caused by both the chemical reactions and the changes deriving mainly from the transformation of milk proteins under the heating temperature effect. Several authors examined the kinetics of these reactions $[3,4]$. This phenomenon cannot be totally removed, but its reduction remains possible [4]. The formation of the fouling of plate heat exchangers PHEs during milk process is affected by several factors such as the $\beta$ lactoglobulin protein $(\beta \mid g)$ formation, milk temperature as well as the PHE geometry $[5,6]$. The formation of the $(\beta \mid \mathrm{g})$ deposit pay the attention of many researchers where their approach is based on the modeling of chemical and microbiological reactions [7-10]. The heat treatment of the milk requires $\mathrm{HE}$ as a chemical reactor and the milk constituents as reagents. Lalande et al., [11] and Petermeier et al., [12] have demonstrated that the thermal denaturation of the $\beta$ lg governed the formation of deposit on the PHE surface towards a temperature of $90^{\circ} \mathrm{C}$. The heat stability of the proteins decreases with decreasing of the $\mathrm{pH}$ [13]. The calcium ions present in the milk influence the $\beta$ Ig denaturation temperature and facilitate the aggregation [14]. The fat presents in the milk has no much effect on the fouling [15]. The prolonged storage of milk at $5^{\circ} \mathrm{C}$ can decrease the fouling due to the action of proteolytic enzymes [16]. Milk preheating can cause denaturation and protein aggregation before the heating section, which then leads to reduce the fouling [17]. This latter can also be reduced by improving the turbulence [4]. The temperature of the milk in the heat exchanger is probably the most important factor to check the fouling [4]. Several studies have examined the characteristics of PHE: Visser and Jeurnink [15] and Yoon and Lund [18] showed that the nature of the surface material (steel ...) as well as the characteristics (surface roughness, doping ...) can influence the fouling. The presence of deposit promotes the adhesion of microorganisms to the surface by various mechanisms, and the deposits provide nutrients for microorganisms to assure their growth [19].

Recently, most researchers used the Computational Fluid Dynamics CFD to solve the heat exchangers equations (LMTD, NTU.) because it is considered as a powerful micro process and a timesaving method especially during design phase have developed a CFD simulation, with the use of the Fluent software [20-25]. The results were applied for the design and commissioning of the HEP. The model allows calculating deposit and removal yield. Also, it allows a realistic distribution of the heat flow along the transfer surface during simulation. Luan and all investigated the reliability of the CFD simulation to study different PHE geometries, in order to improve the mesh quality around the contact points [26]. Recently, Pan and all developed a 2D dynamic model that simulate the fouling layer domain and its effect on the hydrodynamics and heat transfer [27]. Based on these studies, we can conclude that the geometry of the fouling layer plays a vital role in determining the 
hydrodynamics, mass and heat transfer in the PHEs. Most researchers have used the study of flow fields and heat distribution in a rectangular channel heat exchanger. Among them is Ameur [29,30], Georgiadis and Macchietto [31], and Pinson et al., [32].

Therefore, our understanding of milk fouling, especially fouling layer formation and its intricate interactions with transport phenomena and fouling kinetics in the PHEs, is far from complete. There is still an urgent need for more realistic $2 \mathrm{D}$ or $3 \mathrm{D}$ dynamic models that can take into account the domain of fouling layer when simulating milk fouling. For this purpose, a CFD-fluent model has been developed which simulate the fouling thickness and its effect on heat and mass transfer. The paper is arranged as follows. Section 1 describe the fouling mechanism formed on the PHE and the evaluation of the deposition kinetics of the fouling material. Section 2 presents the numerical model to be solved and the and resolution of the conservation equations under transient conditions by adopting a turbulence model in both equations $(k-\varepsilon)$ and quantification of the fouling resistance. Section 3 illustrates the results obtained which is the effect of different the properties (effective thermal conductivity) of the deposit and fibrous to reduce the warm caused by the fouling. In addition, validation of the CFD results with literature results is also shown.

\section{Mechanism of Fouling}

A schematic representation of the proposed fouling model is given in (Figure 1). Native proteins (N) transformed into denatured proteins (D) react according to the first order reaction using the Arrhenius law in order to give protein aggregates (A). Afterward a mass transfer between the fluid volume and the thermal boundary layer occurs for each protein.

Only the aggregated proteins $(A)$ are deposited on the wall at pastorisation temperature. The deposit rate is proportional to the concentration of the aggregated protein in the thermal boundary layer. The fouling resistance is proportional to the thickness of the deposit. Proteins react in the volume of the fluid as well as in the milk boundary layer.

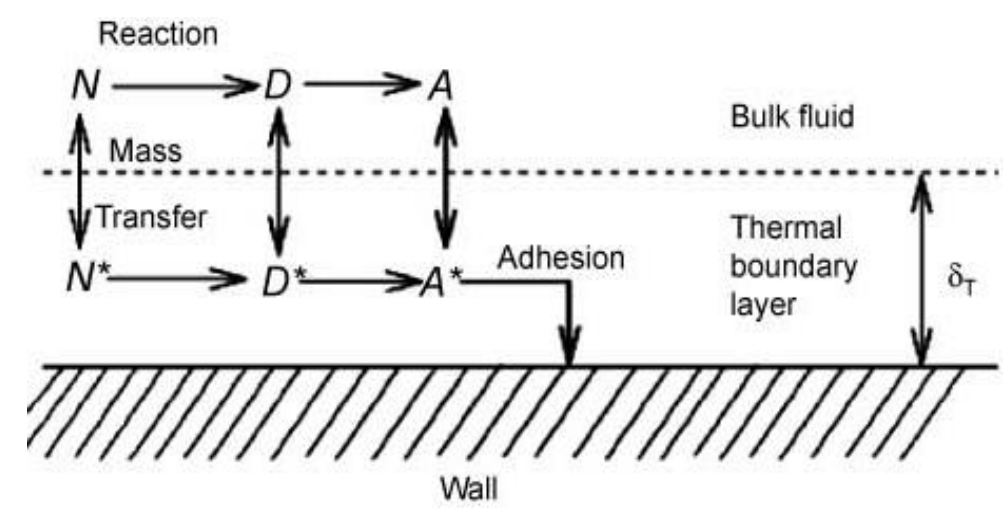

Fig. 1. Model of proteins deposition on the heat exchanger surface [31]

Assuming that the surface of the plate is flat and smooth, the reaction scheme produced during the formation of the fouling by the milk is presented in Figure 2. 


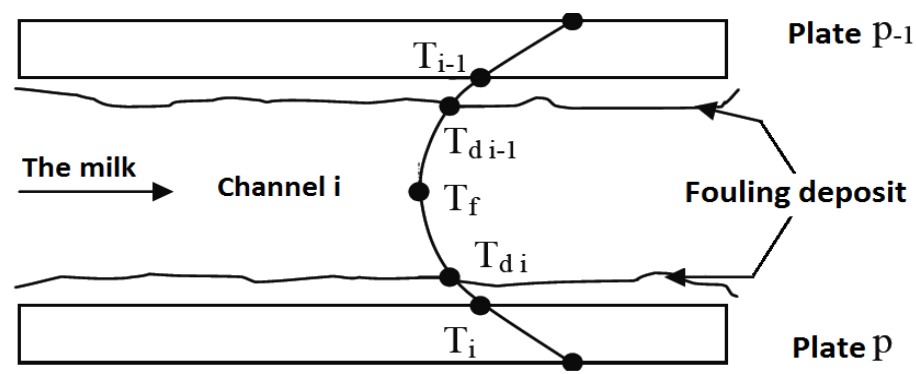

Fig. 2. Distribution of the plates, fluid, deposit and temperature in the channel

The hypotheses for mass balance models according to Georgiadis and Macchietto [31] are:

- Physical properties variation of the milk with the temperature is neglected.

- No diffusion mass transfer is considered in the axial direction.

\section{Mathematical Formulation}

\subsection{Introduction}

The equations of conservation governing the heat transfer in a 2D plane turbulent regime were used with some simplifications. The standard turbulence model $k-\varepsilon$ was used. The mathematical formulation was divided into two parts: The equation model for turbulent flow in a fluid medium, and the equation model in the presence of a porous medium.

In both cases, the conservation equations, the closure model of the equations in turbulent flow and the boundary conditions were presented. Assumptions were considered in order to reduce the mathematical formulation. In this context, the approach to a single domain was employed using a single system of equations governing the whole area studied (fluid and porous).

\subsubsection{Turbulence modelling in a totally fluid medium}

The mass conservation equation is given by:

$$
\frac{\partial U_{i}}{\partial X_{i}}=0
$$

Conservation equation of the amount of movement is given by:

$$
\rho \bar{U}_{j} \frac{\partial \bar{U}_{i}}{\partial X_{j}}=-\frac{\partial \bar{P}}{\partial X_{i}}+\frac{\partial}{\partial X_{j}}\left[\left(\mu+\mu_{t}\right) \frac{\partial \bar{U}_{i}}{\partial X_{j}}\right]+\frac{\partial}{\partial X_{j}} \mu_{t}\left[\frac{\partial \bar{U}_{i}}{\partial X_{j}}\right]
$$

Conservation energy equation is given by:

$$
\rho \bar{U}_{j} \frac{\partial \bar{T}}{\partial X_{j}}=\frac{\partial}{\partial X_{j}}\left(\frac{\mu}{P_{r}}+\frac{\mu_{t}}{\sigma_{t}}\right) \frac{\partial \bar{T}}{\partial X_{j}}
$$


General equation of the turbulent kinetic energy (k):

$\rho \bar{U}_{j} \frac{\partial k}{\partial X_{j}}=\frac{\partial}{\partial X_{j}}\left[\left(\mu+\frac{\mu_{t}}{\sigma_{k}}\right) \frac{\partial k}{\partial X_{j}}\right]+\mu_{t} \frac{\partial \overline{U_{i}}}{\partial X_{j}}\left(\frac{\partial \overline{U_{i}}}{\partial X_{j}}+\frac{\partial \overline{U_{j}}}{\partial X_{i}}\right)-\rho \varepsilon$

Transport equation for the turbulent dissipation rate $(\varepsilon)$ is given by:

$\rho \bar{U}_{j} \frac{\partial \varepsilon}{\partial X_{j}}=\frac{\partial}{\partial X_{j}}\left[\left(\mu+\frac{\mu_{t}}{\sigma_{\varepsilon}}\right) \frac{\partial \varepsilon}{\partial X_{j}}\right]+C_{\varepsilon 1} \frac{\varepsilon}{k} \mu_{t} \frac{\partial \overline{U_{i}}}{\partial X_{j}}\left(\frac{\partial \overline{U_{i}}}{\partial X_{j}}+\frac{\partial \overline{U_{j}}}{\partial X_{i}}\right)-C_{\varepsilon 2} \rho \frac{\varepsilon^{2}}{k}$

\subsubsection{General model and turbulence modelling in porous medium}

The equations of continuity, movement, energy, turbulent kinetic energy and turbulent dissipation were written in index form in the following equations system [33]:

$$
\frac{\partial \overline{U_{i}}}{\partial X_{i}}=0
$$

$$
\begin{aligned}
& U_{j} \frac{\partial U_{i}}{\partial X_{j}}=-\frac{1}{\rho} \frac{\partial P}{\partial X_{i}}+\frac{\partial}{\partial X_{i}}\left[\left(v J+v_{t}\right) \frac{\partial \bar{U}_{i}}{\partial X_{j}}\right]+\frac{\partial}{\partial X_{i}}\left[v_{t} \frac{\partial \bar{U}_{i}}{\partial X_{j}}\right]-\frac{2}{3} \frac{\partial k}{\partial X_{j}}-\phi \frac{v}{K} U_{i}-\phi^{2} \frac{C_{F}}{K^{1 / 2}} \\
& {\left[\left(U_{j} U_{j}\right)^{1 / 2} U_{i}-\frac{U_{j}}{\left(U_{j} U_{j}\right)^{1 / 2}}\left(v_{t}\left\{\frac{\partial U_{i}}{\partial X_{j}}+\frac{\partial U_{j}}{\partial X_{i}}\right\}-\frac{2}{3} k\right)\right]}
\end{aligned}
$$

$\phi \cdot U_{j} \frac{\partial T}{\partial X_{j}}=\frac{\partial}{\partial X_{i}}\left[\left(\frac{\lambda_{e f f}}{\left(\rho \cdot C_{p}\right)_{f}}+\phi \frac{v_{t}}{\sigma_{t}}\right) \frac{\partial T}{\partial X_{j}}\right]$

$\bar{U}_{j} \frac{\partial k}{\partial X_{j}}=v_{t}\left(\frac{\partial \bar{U}_{i}}{\partial X_{j}}+\frac{\partial \overline{U_{j}}}{\partial X_{i}}\right) \frac{\partial \bar{U}_{i}}{\partial X_{j}}+\frac{\partial}{\partial X_{k}}\left[v J+\frac{v_{t}}{\sigma_{t}}\right] \frac{\partial k}{\partial X_{k}}-2 \phi \frac{v}{K} k-J \varepsilon-\phi^{2} \frac{C_{F}}{\left(U_{k} U_{k}\right)^{1 / 2} K^{1 / 2}}$

$$
\left[\begin{array}{l}
-v_{t}\left(\frac{\partial U_{i}}{\partial X_{k}}+\frac{\partial U_{k}}{\partial X_{i}}\right)\left(U_{k} U_{j}\right)+\frac{8}{3} k\left(U_{k} U_{k}\right)+\frac{5}{3}\left(-\frac{4}{3} C_{s} U_{k} \frac{k^{2}}{\varepsilon} \frac{\partial k}{\partial X_{k}}\right) \\
+\frac{4}{3} C_{s} U_{k} \frac{k^{2}}{\varepsilon} v_{t} \frac{\partial}{\partial X_{i}}\left(\frac{\partial U_{i}}{\partial X_{k}}+\frac{\partial U_{k}}{\partial X_{i}}\right)
\end{array}\right]
$$




$$
\begin{aligned}
& \bar{U}_{k} \frac{\partial \varepsilon}{\partial X_{k}}=\frac{\partial}{\partial X_{k}}\left[\left(v J+\frac{v_{t}}{\sigma_{\varepsilon}}\right) \frac{\partial \varepsilon}{\partial X_{k}}\right]+C_{\varepsilon 1} \frac{\varepsilon}{k} v_{t}\left(\frac{\partial U_{i}}{\partial X_{j}}+\frac{\partial U_{j}}{\partial X_{i}}\right) \frac{\partial U_{i}}{\partial X_{j}}-J C_{\varepsilon 2} \frac{\varepsilon^{2}}{k}-2 \phi \frac{v}{K} \varepsilon-2 \phi^{2} \frac{C_{F}}{K^{1 / 2}} \\
& {\left[\begin{array}{l}
\left(U_{k} U_{k}\right)^{1 / 2} \varepsilon+v\left(\frac{\partial}{\partial X_{k}}\left(\left(U_{k} U_{k}\right)^{1 / 2}\right)\right) \frac{\partial k}{\partial X_{j}}+\frac{v}{3} \frac{\partial}{\partial X_{k}} \frac{\left(U_{k} U_{i}\right)}{\left(U_{k} U_{k}\right)^{1 / 2}} \frac{\partial k}{\partial X_{j}}+ \\
\frac{U_{k}}{\left(U_{k} U_{k}\right)^{1 / 2}} C_{\varepsilon 3} \frac{k}{\varepsilon} v_{t}\left(\frac{\partial U_{k}}{\partial X_{j}}+\frac{\partial U_{j}}{\partial X_{k}}\right) \frac{\partial \varepsilon}{\partial X_{j}}+\frac{1}{3} \frac{U_{k} U_{i}}{\left(U_{k} U_{k}\right)^{1 / 2} \varepsilon-} \\
\frac{v}{2} v_{t} \frac{\partial}{\partial X_{j}} \frac{\left(U_{k} U_{i}\right)}{\left(U_{k} U_{k}\right)^{1 / 2}}\left(\frac{\partial^{2} U_{i}}{\partial X_{j} \partial X_{k}}+\frac{\partial^{2} U_{k}}{\partial X_{j} \partial X_{i}}\right)-\left(\frac{2}{3} \frac{U_{k}}{\left(U_{k} U_{k}\right)^{1 / 2}} C_{\varepsilon 3} \frac{k}{\varepsilon} k\right) \frac{\partial \varepsilon}{\partial X_{j}}
\end{array}\right]}
\end{aligned}
$$

In this model of turbulence, the values of the most common constants are:

$\mathrm{C} \varepsilon 1=1.44 ; \mathrm{C} \varepsilon 2=1.9 ; \sigma \mathrm{k}=1$ et $\sigma \varepsilon=1.2$

Boundary conditions:

The following conditions were considered:

\section{Dynamic field:}

At the inlet a constant rate profile:

$\mathrm{x}=0: \mathrm{u}=\mathrm{u}_{\mathrm{e}}$ and $\mathrm{v}=0$

Adhesion condition at the wall:

$y=0$ and $y=H: u=v=0$

At the outlet a dynamic regime was established:

$\mathrm{x}=\mathrm{L}: \frac{\partial \mathrm{u}}{\partial \mathrm{x}} \mid=0$

Temperature field:

A constant temperature was imposed on walls:

$y=0$ and $y=H: T=T_{p}=400 K$

A constant temperature at the inlet:

$\mathrm{x}=0: \mathrm{T}=\mathrm{T}_{\mathrm{e}}=300 \mathrm{~K}$

Negligible axial diffusion in the output:

$\mathrm{X}=\mathrm{L}:\left.\frac{\partial T}{\partial x}\right|_{x=L}=0$ 
Turbulent kinetic energy and turbulent dissipation:

Adhesion condition at the wall:

$\mathrm{y}=0$ and $\mathrm{y}=\mathrm{H}: \mathrm{k}=0$ and $\varepsilon=v \frac{\partial^{2} \mathrm{k}}{\partial \mathrm{y}^{2}}$

At the inlet:

$\mathrm{X}=0: \mathrm{k}=\mathrm{k}_{\mathrm{e}}=\mathrm{IU} \mathrm{e}_{\mathrm{e}}^{2}$ and $\varepsilon=\varepsilon_{\mathrm{e}}=v \frac{\mathrm{k}_{\mathrm{e}}^{\frac{3}{2}}}{\alpha \mathrm{D}_{\mathrm{h}}}$

Where:

I: turbulence rate, varied between $0.1 \%$ à $10 \%$ and $\alpha=0.33$. At the output an established regime was required, as follows:

$\mathrm{x}=\mathrm{L}:\left.\frac{\partial k}{\partial x}\right|_{x=L}=\left.\frac{\partial \varepsilon}{\partial x}\right|_{x=L}=0$

Conditions at the fluid interface - porous:

The approach in a single area in the formulation of the conservation equations allows describing simultaneously both of flows and transfers in fluid and porous areas. The conditions of linking at the interface were then checked implicitly.

The passage from the fluid domain to porous medium was made by a variation of permeability and porosity. With the approach in a single domain, the following limit values of the appropriate parameters to the porous medium allow to define the type of the modeled medium:

Fluid medium: $\mathrm{J}=1, \mathrm{R}_{\mathrm{c}}=1, \phi=1, \mathrm{D}_{\mathrm{a}} \rightarrow \infty$

Porous medium: $\mathrm{J} \geq 1, \mathrm{R}_{\mathrm{c}} \neq 1, \phi \prec 1$, finite and very low. Where: $\mathrm{R}_{\mathrm{c}}=\frac{\lambda_{\text {eff }}}{\lambda}$ and $\mathrm{K}=\mathrm{D}_{\mathrm{a}}$.

\section{Numerical Resolution}

\subsection{Introduction}

The equations of the system were nonlinear partial derivatives of differential equations, elliptical and coupled. Because of their complexity, these equations were solved using a numerical method. The numerical procedure adopted was based on the finite volume method on the Fluent CFD software.

\subsection{Resolution Procedure}

The resolution process was composed, as any type of CFD software, of three key elements which were: the preprocessor, the solver and postprocessor. Thus, the implementation of the numerical simulation was performed. There are several types of structured, unstructured or hybrid meshing. A structured meshing is generally composed of quadrilateral meshes in two dimensions (2D or surface meshing) and hexahedral in three-dimensional (3D or volume meshing). Whereas an unstructured 
meshing is composed of quadrilateral or triangular meshes in 2D and 3D tetrahedral or hexahedral, in a hybrid meshing, the meshes near the walls are quadrilaterals in $2 D$ and $3 D$ hexahedral and the meshes of the rest of the field are triangles in 2D and tetrahedral in 3D. In the present work we have considered the quadrilateral mesh represented in (Figure 3):

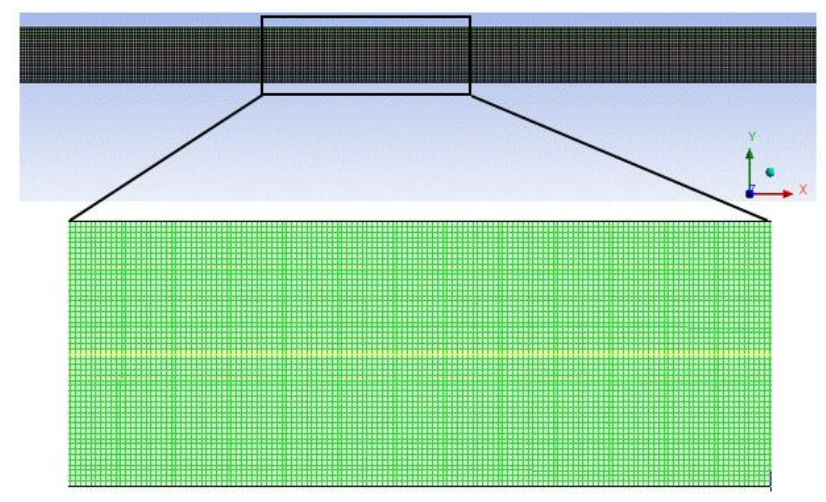

Fig. 3. Meshing of the channel

The code employed uses two types of solvers. The choice of the solver depends mainly on the nature of the flow (compressible or incompressible, stationary or non-stationary).

The distinct and coupled approaches differ in the way, continuity, and (if applicable) the energy and the species of equations to be solved: the different solver solves the equations sequentially, while the coupled solver solves them simultaneously. Both formulations were used to solve the scalar equations of a turbulent model of the milk flow. Finally, the boundary conditions were defined as follows:

- The two fluids at the inlet;

- The walls;

- The two fluids at the outlet;

- The axis of symmetry.

\section{Results and Discussion}

\subsection{Introduction}

The milk fouling in the PHE has been considered for turbulent flows completely developed by evaluating and comparing the various macroscopic flows, such as those of dispersion and diffusion (Figure 4). The simulations concern the influence of the variation of the geometrical and thermophysical parameters of the deposit, the dynamic and the temperature field. The various data are indicated in Table 1.

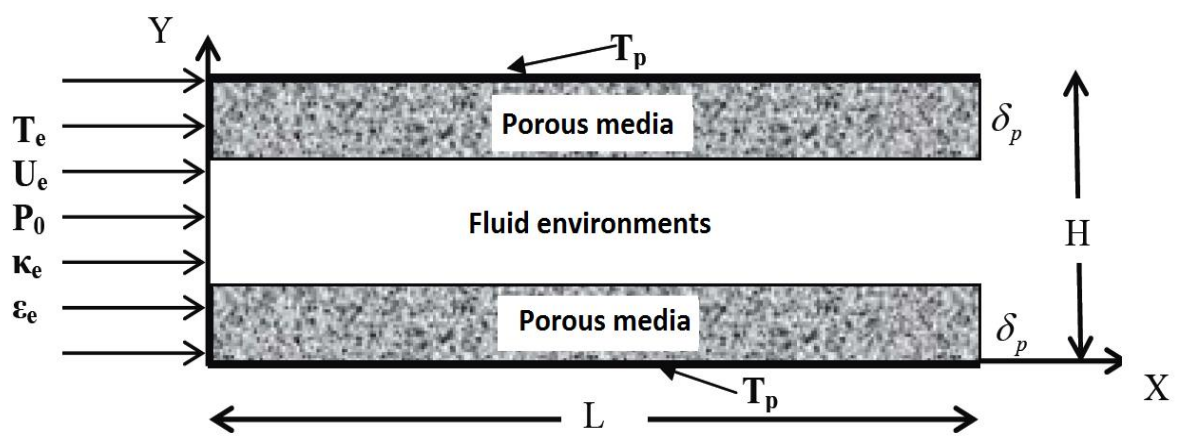

Fig. 4. Flow in a channel of rectangular section 
Table 1

Dimensional variables and physicochemical parameters

\begin{tabular}{lll}
\hline Parameter & Symbol & Value \\
\hline Plate temperature $(\mathrm{K})$ & $\mathrm{T}_{\mathrm{p}}$ & 400 \\
Temperature of the Milk (K) & $\mathrm{T}_{\mathrm{e}}$ & 300 \\
Milk velocity $(\mathrm{m} / \mathrm{s})$ & $\mathrm{V}_{\mathrm{f}}$ & 0.6 \\
Length of the pipe $(\mathrm{m})$ & $\mathrm{L}$ & 4 \\
Distance between the plates $(\mathrm{m})$ & $\mathrm{H}$ & 0.02 \\
Thermal conductivity of milk $\left(\mathrm{w} / \mathrm{m} .{ }^{\circ} \mathrm{C}\right)$ & $\lambda_{\mathrm{f}}$ & 16.3 \\
Viscosity of milk $(\mathrm{kg} / \mathrm{m} . \mathrm{s})$ & $\mu_{\mathrm{f}}$ & $6.04310^{-4}$ \\
Density of milk $(\mathrm{kg} / \mathrm{m} 3)$ & $\rho_{\mathrm{f}}$ & 1033 \\
Thermal capacity of the milk (KJ / kg.K) & $\mathrm{Cp}_{\mathrm{f}}$ & 1921 \\
\hline
\end{tabular}

\subsection{Meshing Sensibility}

The stability calculations depend on the choice of the meshing for a code based on the employment of the finite volume. Several trials were realized. The effect of the deposit thickness of $0.0018 \mathrm{~mm}$ for three meshing $(\Delta x=\Delta y=$ Mesh1 $=0.0008$, Mesh2 $=0.00085$, Mesh3 $=0.00075)$ on the temperature field in the middle of the channel is displayed in Figure $5(\mathrm{a})$ and The Nusselt variation on the channel walls is illustrated Figure $5(b)$. A good stability of the calculation was observed with three grids. The average error between the different grids did not exceed $1 \%$.

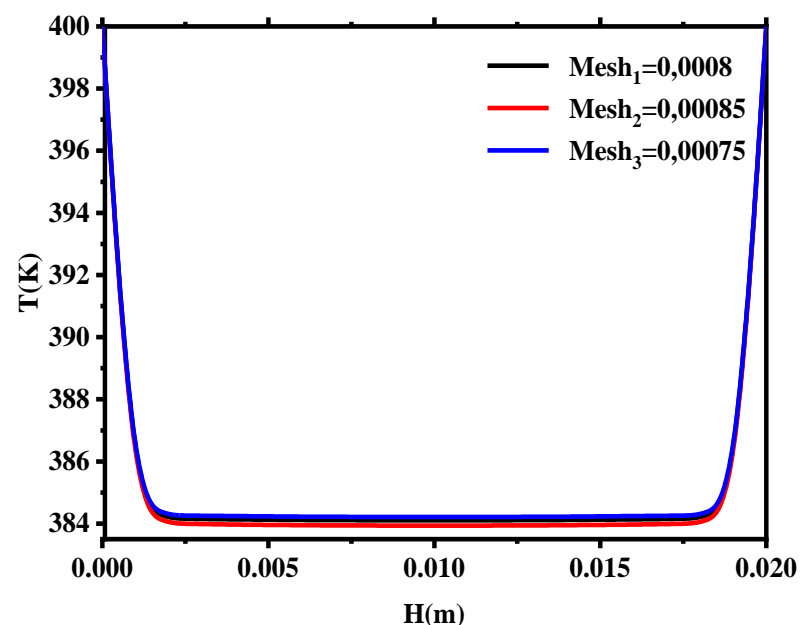

(a)

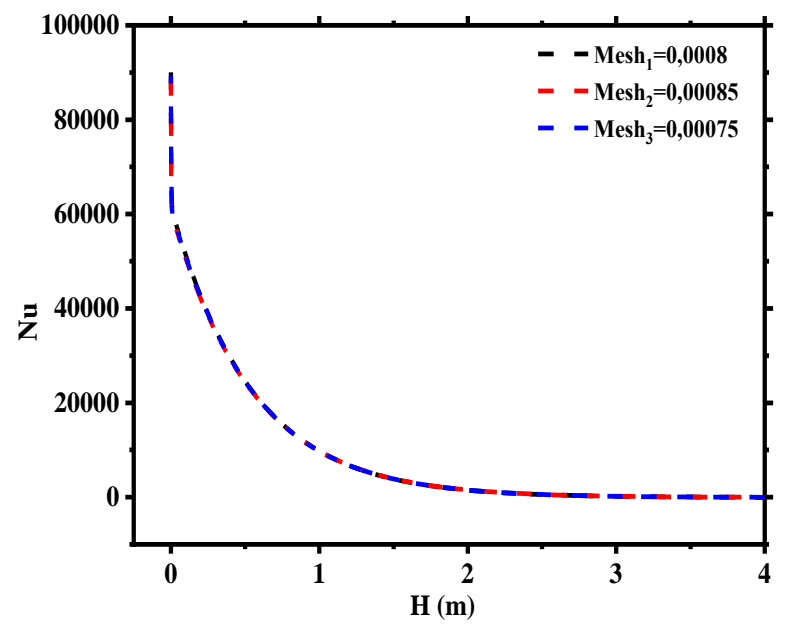

(b)

Fig. 5. The effect of the mesh on the variation of the (a): temperature and the (b): Nusselt number for different meshes

\subsection{Model Validation}

Model validation has been done on a comparison with the work of Pinson et al., [32] and Sadouk [33]. They studied the turbulent flow model between two parallel plates using the closure model k$\varepsilon$. In this context, the evolution of the rate profile in the fully developed region of the channel, for various Reynolds number based on the hydraulic diameter of the pipe has been considered. The purpose was to study the effect of the variation of the Reynolds number on the rate profile. Figure 6 shows, for various Reynolds numbers, the profiles of the longitudinal rate ratio at the input rate $U_{0}$. Figure 6 presents the validation of the present work results with Sadouk [33].

While Figure 6(b) illustrates the validation with Pinson et al., [32]. From Figure 6, a good agreement results were obtained for the longitudinal velocity profile, established in dynamic 
conditions. The comparison was based on the dimensionless variation of the fluid velocity. For low Reynolds numbers, the turbulent boundary layer strongly affects the velocity profile in the center of the flow. The thickness of the boundary layer at the walls evolved significantly between $\operatorname{Re}=10^{4}$ and $\operatorname{Re}=10^{5}$.

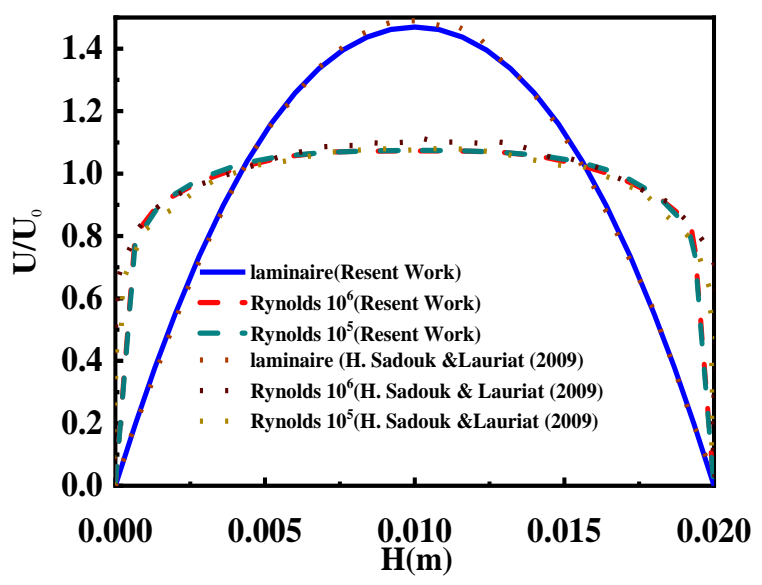

(a)

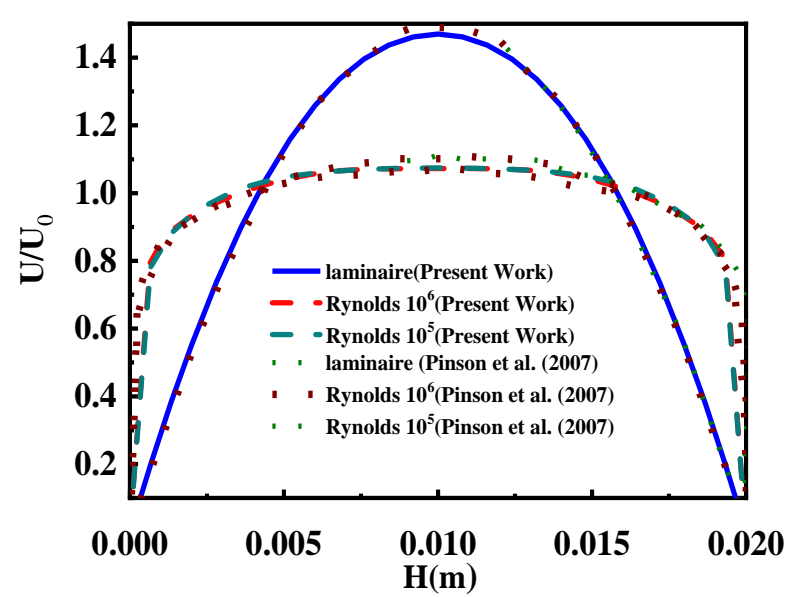

(b)

Fig. 6. Dimensionless velocity profile for different Reynolds numbers (laminar; $10^{5} ; 10^{6}$ ) for validation of the present work results with (a) Sadouk [33] and (b) Pinson et al., [32]

\subsection{Dynamic Study}

\subsubsection{Speed field}

Figure 7 represents the evolution of the temperature at the outlet of the HE for different Reynolds number. The increase of the Reynolds number reduced the temperature in the channel. This can be explained by the fact that with the increase of the Reynolds number therefore an increase in the velocity, the fluid stayed less time in the channel, leading to a lower heat exchange and a smaller increase in the temperature.

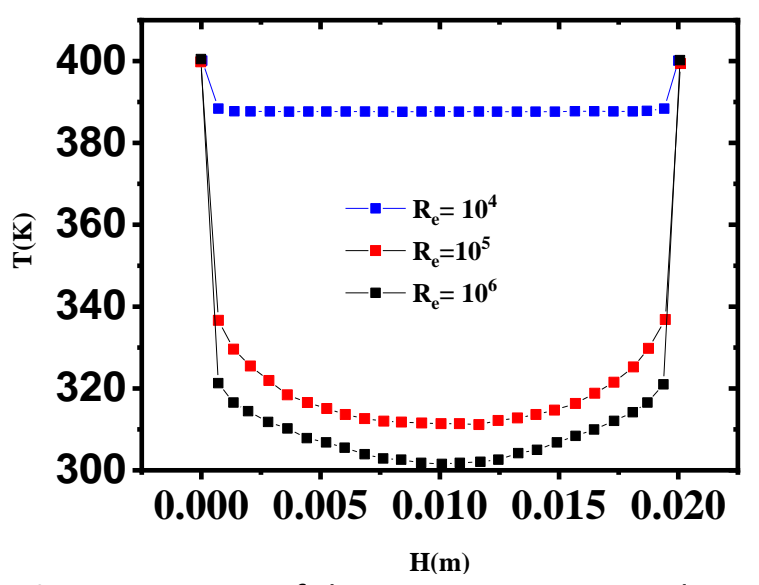

Fig. 7. Variation of the temperature according to channel medium for different Reynolds number

The evolution of the speed field at the outlet of the PHE for different thicknesses of the porous medium is shown in Figure 8. The rate decreased with the increase of the thickness of the porous layer, since. T the latter reduced the section of passage of the fluid. It can be seen in Figure 8 that the 
velocity field increases rapidly as the thickness increases and becomes lower at steady state for a pipe without porous layers. The velocity vectors at the exit of the porous medium are more important than the vectors in the fluid part for the two cases (convection and the imposed temperature). It can be seen that, in the case of a channel with a porous deposit on the walls, the shape of the velocity profile has a low gradient at the outlet of the channel at the fluid level, whereas the porous medium outlets where it is reached is maximum. The reduction of the fluid flow cross-section from the increase in the thickness of the porous medium causes $(e=0.1$ and 0.4$)$ an acceleration of the velocity in the channel outlet, whereby the maximum velocity at the outlet increases as the thickness of the porous medium increases.
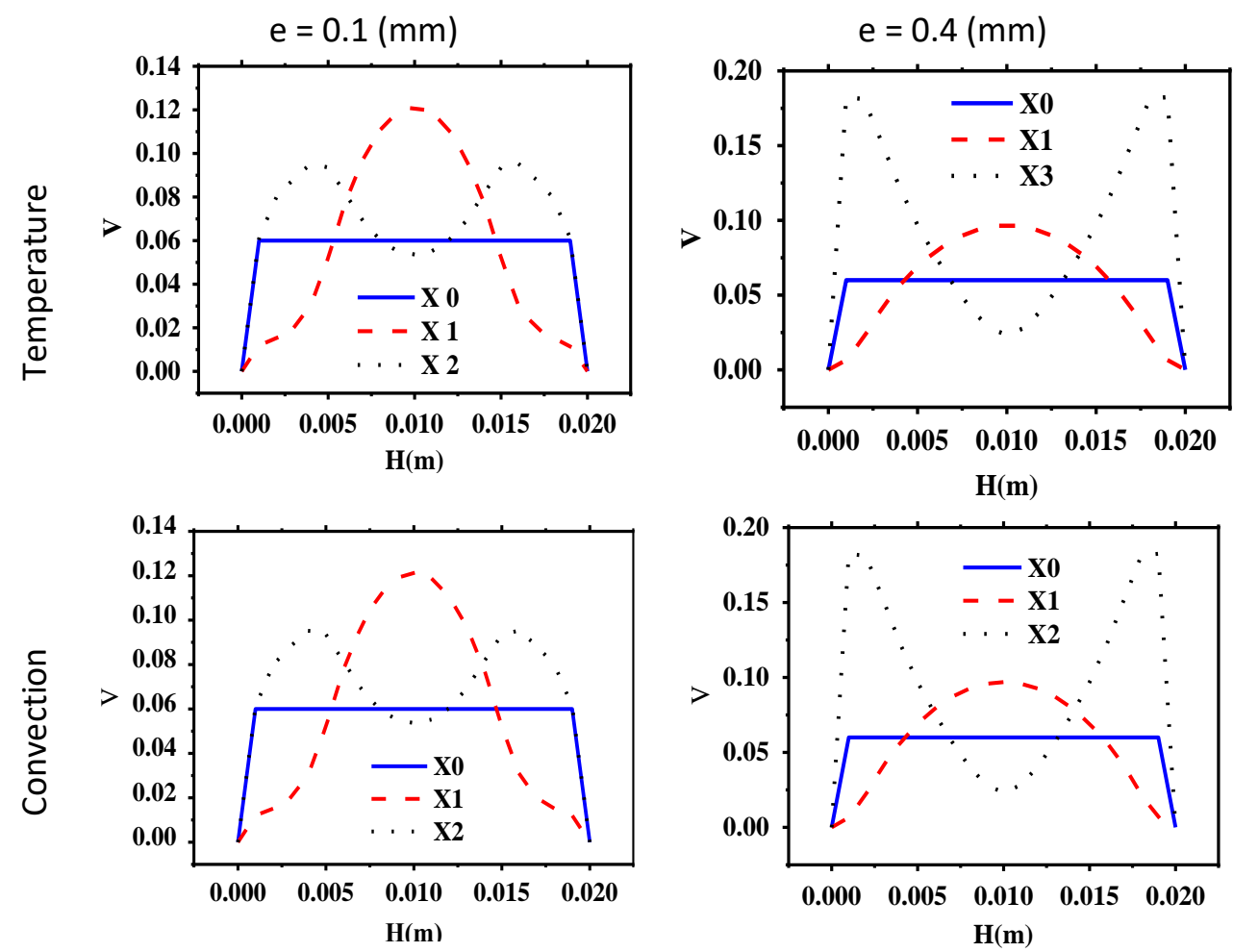

Fig. 8. Speed vector at the outlet of the channel

\subsubsection{Outline of the turbulent kinetic energy $k$}

Figure 9 highlights the evolution of the turbulent kinetic energy at the output of the channel. It reached a maximum value at the fluid-wall and / or the fluid-deposit interface. The reduction of the fluid passage section over both time and nature of the contact interface (fluid-deposit) caused turbulent energy peak.

At this interface, in addition to the resistance due to the viscous and inertial forces, an important additional resistance is due to terms of Darcy and Forchheimer, leading to a further acceleration towards the centre of the channel; turbulent kinetic energy decreased gradually and reached its minimum in the central section of the channel. The reduction of the fluid passage section increased the kinetic energy. It was almost null in the deposit area.

\subsubsection{Profile of epsilon turbulent dissipation}

Figure 10 illustrates the distribution of the turbulent kinetic energy dissipation rate on the channel output. The profiles of turbulent kinetic energy and turbulent dissipation varied in the same 
way. A maximum dissipation rates was initially observed at the fluid-wall interface, which was more important at the fluid-deposit interface. It can be noticed that the turbulent dissipation rate $\varepsilon$ was important in the porous region and increased gradually along the channel, reaching its maximum value at the channel wall. It can also be noticed that epsilon $(\varepsilon)$ maximum increased when the thickness of the porous medium decreased; this dissipation is generally near the walls.
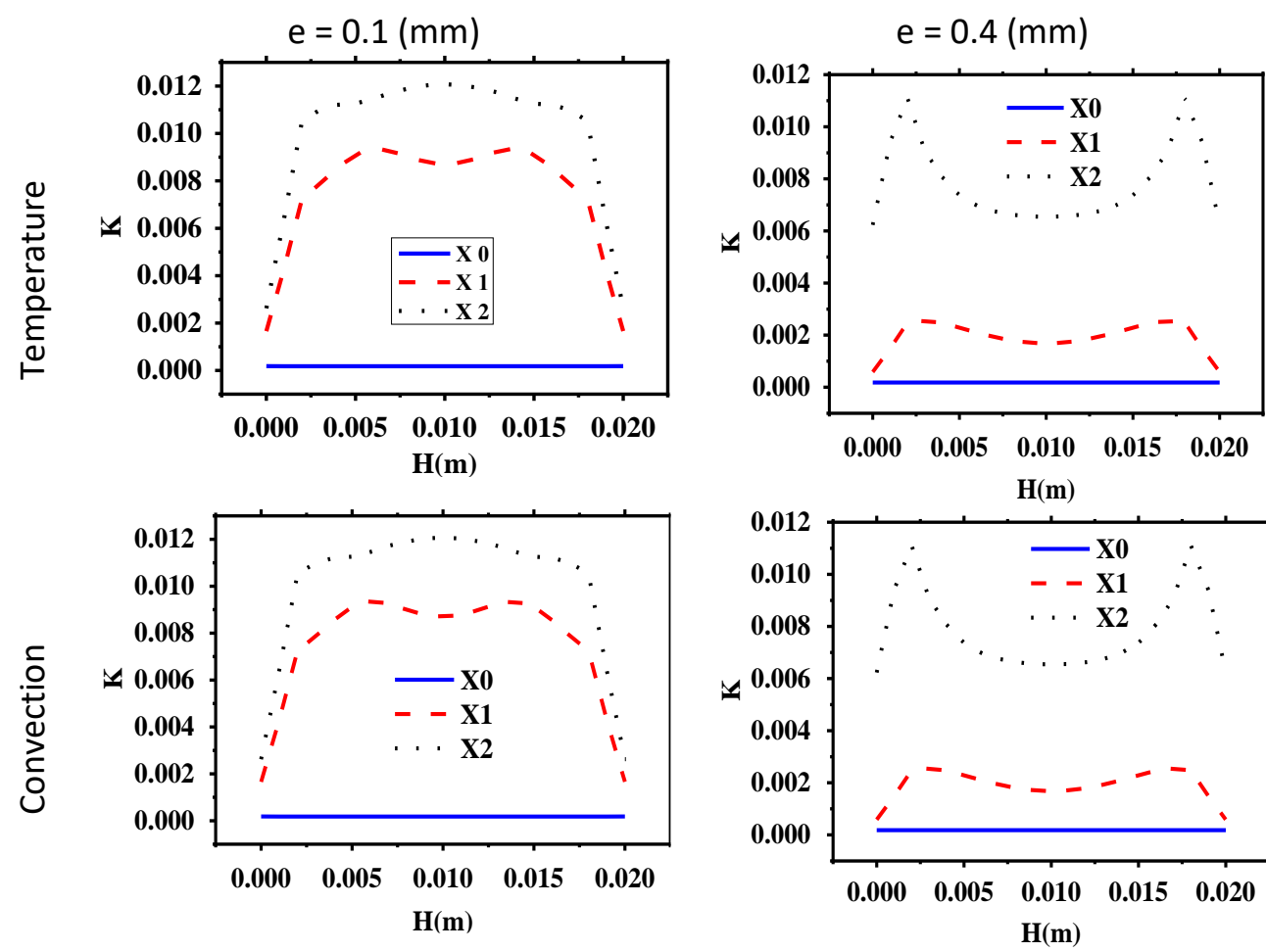

Fig. 9. The contour of the turbulent kinetic energy $k$ at the inlet of the channel
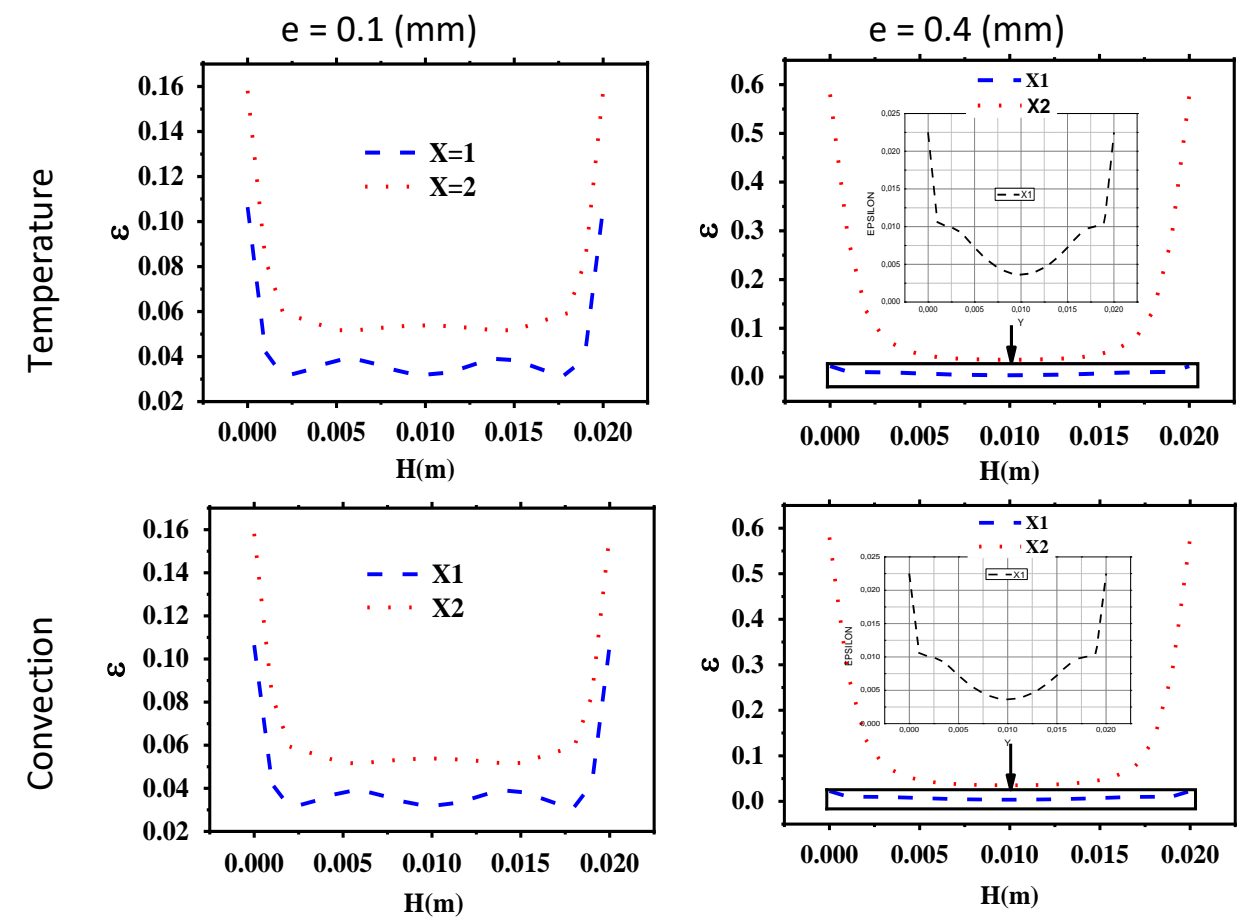

Fig. 10. The distribution of turbulent dissipation rate $(\varepsilon)$ along $y$ at the output exchanger for different thicknesses of the porous medium 


\subsection{Thermal Study}

\subsubsection{Variation of Nusselt number}

The evolution of the Nusselt number according to the position is represented in the Figure 11. A fall of the Nusselt number along the channel indicates a degradation of the quality of heat exchange with the warm fluid due to the insulating quality of the deposit $\left(\lambda=0.5 \mathrm{w} / \mathrm{m} .{ }^{\circ} \mathrm{C}\right)$ dominates increase flow boundary layer [34].

\subsubsection{Temperature profile}

The evolutions of the temperature storyteller at the entrance of the channel, the temperature in the channel without deposit and the temperature according to $Y$ direction in the medium of the channel are shown in Figure 12, Figure 13, respectively.

A gradual increase of the temperature in the channel reaches a maximum value of $T=400 \mathrm{~K}$ after the medium of the channel was observed because of the heat exchange without the presence of deposit. By contrast, during the deposit, the resistance to heat transfer increased gradually and the fluid was heated less and less due the deposit was installed.

A high amount of heat was stored in the porous matrix. The fouling deposit constitutes a thermally insulating layer which reduced the heat transfer.
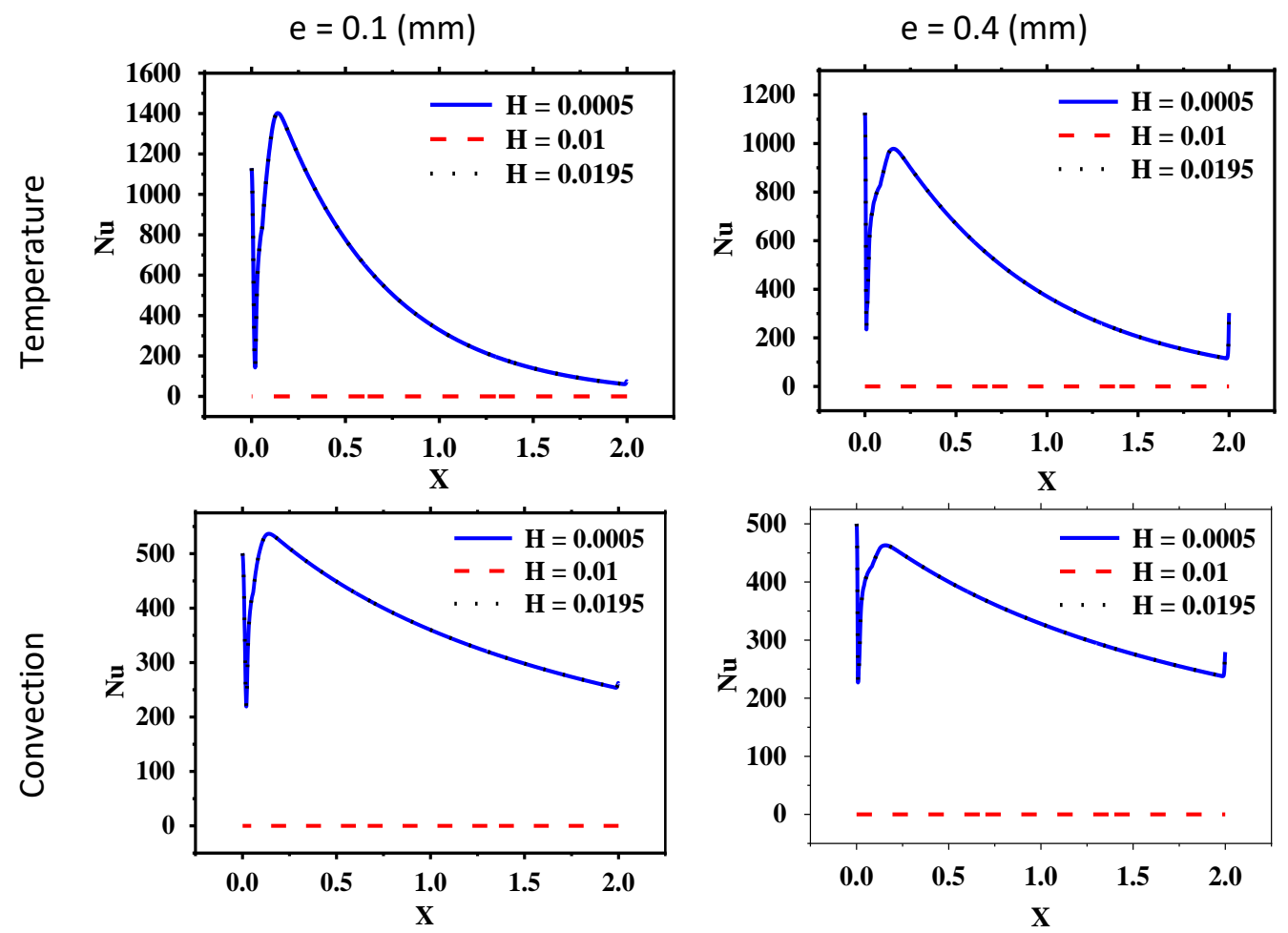

Fig. 11. Variation of the Nusselt number along $x$ at the outlet of the exchanger 

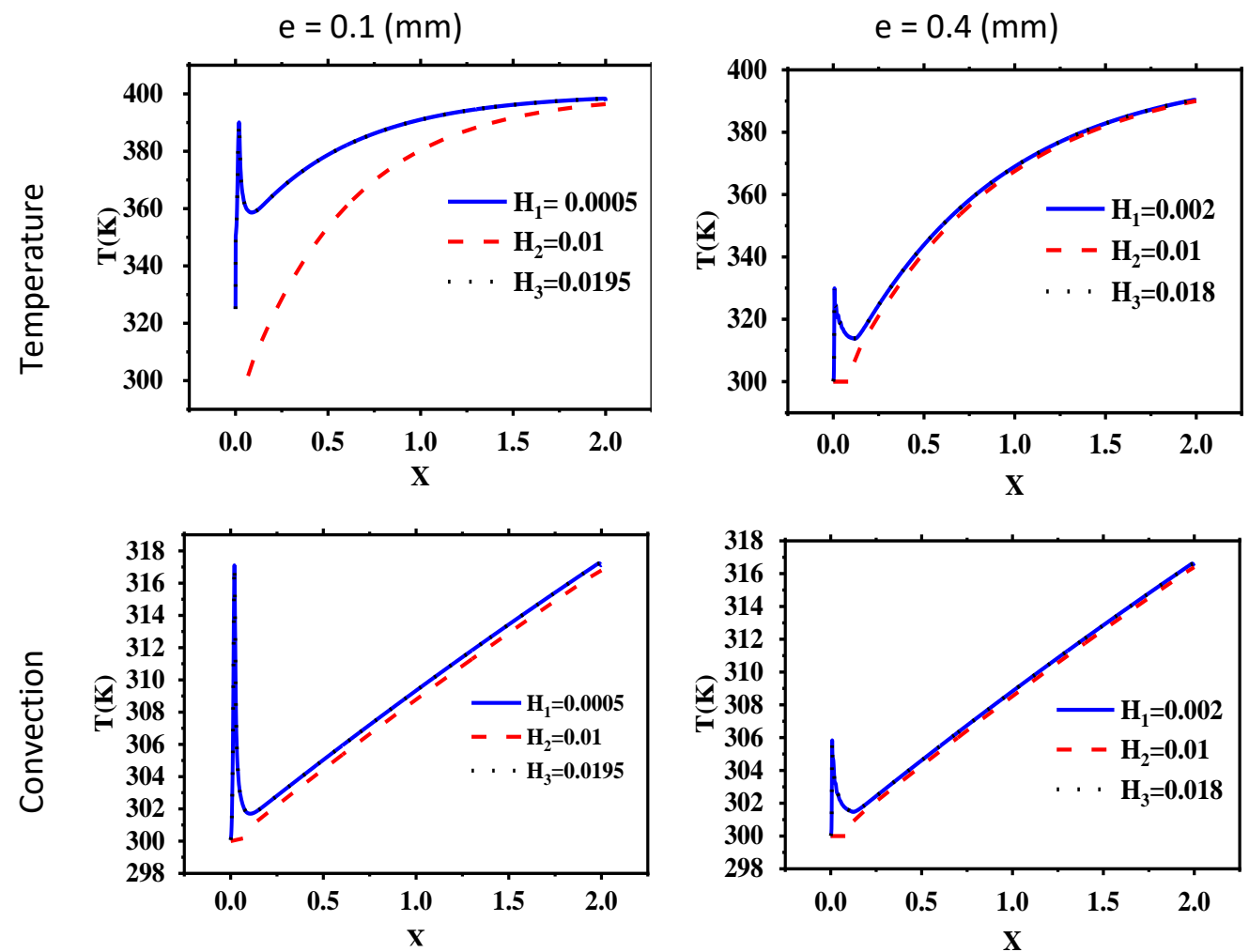

Fig. 12. Variation of the temperature at different point in the medium of the channel during the deposition
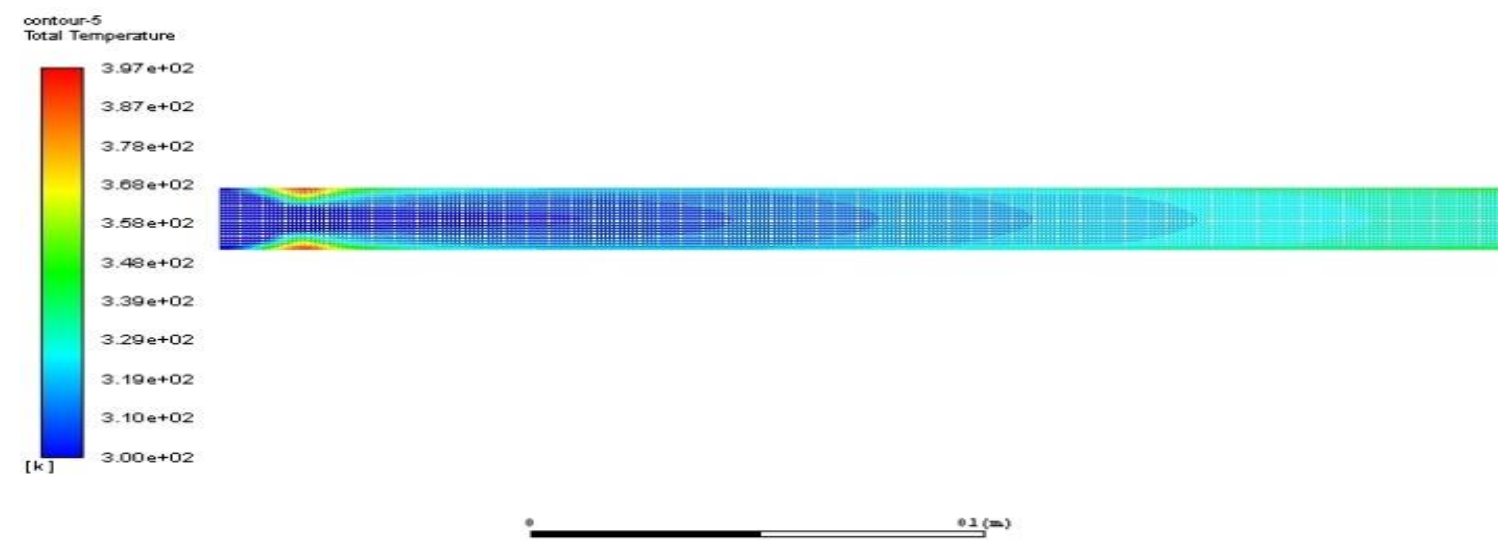

(a) Temperature

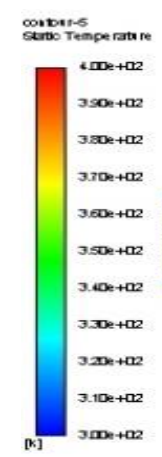

(b) Convection

Fig. 13. The temperature storyteller at the inlet of the channel for these two cases (a) and (b) 


\section{Conclusion}

The fouling in plate heat exchangers upon heating milk using Fluent software has been considered based on thermal energy by visualizing the speed fields and the temperature distribution in the plate heat exchanger channel particularly in turbulent regime.

In this context, the considered physical model consisted of a constant flow rate between two parallel flat plates with imposed temperature. A mathematical model in steady state has been established at the macroscopic scale for a turbulent flow between two flat plates in the presence of a porous medium.

The closure $k-\varepsilon$ model has been chosen and the conservation equations for the specific conditions for the physical model have been developed. The adopted numerical procedure was based on the finite volume method on which the Fluent software was also based.

The validation of the model in the case of a laminar flow regime with a porous medium and then in turbulent regime has been shown by the ability of the established code to set the captured dynamic and thermal aspects due to the fouling.

The results show:

i. A maximum speed at the outlet of the PHE increased when the thickness of the porous medium increased.

ii. An increase in the average temperature over the whole length of the PHE channel during fouling deposition.

iii. A gradual drop in the average temperature at the outlet of the channel at the fluid level due to the decrease of the Reynolds number.

iv. A fouling deposit caused effects of friction and a greater additional thermal resistance.

v. High reduction of the temperature gradient and the Nusselt number in the channel medium.

\section{References}

[1] Bott, Theodore Reginald. Fouling of heat exchangers. Elsevier, 1995. https://doi.org/10.1016/B978-044482186$\underline{7 / 50017-8}$

[2] Taborek, J. "Fouling: The major unsolved problem in heat transfer." Chem. Eng. Prog. 68 (1972): 59-67.

[3] Bansal, Bipan, and Xiao Dong Chen. "A critical review of milk fouling in heat exchangers." Comprehensive Reviews in Food Science and Food Safety 5, no. 2 (2006): 27-33. https://doi.org/10.1111/j.1541-4337.2006.tb00080.x

[4] Belmar-Beiny, M. T., S. M. Gotham, W. R. Paterson, P. J. Fryer, and A. M. Pritchard. "The effect of Reynolds number and fluid temperature in whey protein fouling." Journal of Food Engineering 19, no. 2 (1993): 119-139. https://doi.org/10.1016/0260-8774(93)90038-L

[5] Guerrero-Navarro, A. E., A. G. Ríos-Castillo, C. Ripolles Avila, A. S. Hascoët, X. Felipe, and J. J. Rodriguez Jerez. "Development of a dairy fouling model to assess the efficacy of cleaning procedures using alkaline and enzymatic products." LWT 106 (2019): 44-49. https://doi.org/10.1016/j.Iwt.2019.02.057

[6] Schnöing, Lukas, Wolfgang Augustin, and Stephan Scholl. "Fouling mitigation in food processes by modification of heat transfer surfaces: A review." Food and Bioproducts Processing 121 (2020): 1-19. https://doi.org/10.1016/j.fbp.2020.01.013

[7] Jong, P. De, S. Bouman, and H. J. L. J. Van der Linden. "Original Papers and Proceedings: Fouling of heat treatment equipment in relation to the denaturation of $\beta$-lactoglobulin." International Journal of Dairy Technology 45, no. 1 (1992): 3-8. https://doi.org/10.1111/i.1471-0307.1992.tb01715.x

[8] Ltoyoda, P. J. R. Schreier, and P. J. Fryer. "A computational model for reaction fouling from whey protein solutions." EUR (Luxembourg) (1996): 222-229.

[9] Aouanouk, Saadi Amine, A. Mouheb, Rafik Absi, and R. Zazoun. "The behavior of ß-lactoglobulin protein in plate heat exchanger's channel during milk heat treatment." Acta Alimentaria 46, no. 4 (2017): $411-419$. https://doi.org/10.1556/066.2017.0002

[10] Truong, Tuan H., Ken Kirkpatrick, and Skelte G. Anema. "Role of $\beta$-lactoglobulin in the fouling of stainless steel surfaces by heated milk." International Dairy Journal $66 \quad$ (2017): 18-26. https://doi.org/10.1016/i.idairyj.2016.10.007 
[11] Lalande, Marc, and Jean-Pierre Tissier. "Fouling of heat transfer surfaces related to $\beta$-lactoglobulin denaturation during heat processing of milk." Biotechnology Progress 1, no. $2 \quad$ (1985): $131-139$. https://doi.org/10.1002/btpr.5420010210

[12] Petermeier, H., R. Benning, A. Delgado, U. Kulozik, J. Hinrichs, and T. Becker. "Hybrid model of the fouling process in tubular heat exchangers for the dairy industry." Journal of Food Engineering 55, no. 1 (2002): 9-17. https://doi.org/10.1016/S0260-8774(01)00223-0

[13] Foster, Carole L., Michel Britten, and Margaret L. Green. "A model heat-exchange apparatus for the investigation of fouling of stainless steel surfaces by milk I. Deposit formation at $100^{\circ} \mathrm{C}$." Journal of Dairy Research 56 , no. 2 (1989): 201-209. https://doi.org/10.1017/S002202990002642X

[14] Christian, G. K., S. D. Changani, and P. J. Fryer. "The effect of adding minerals on fouling from whey protein concentrate: development of a model fouling fluid for a plate heat exchanger." Food and Bioproducts Processing 80, no. 4 (2002): 231-239. https://doi.org/10.1205/096030802321154718

[15] Visser, J., and Th JM Jeurnink. "Fouling of heat exchangers in the dairy industry." Experimental Thermal and Fluid Science 14, no. 4 (1997): 407-424. https://doi.org/10.1016/S0894-1777(96)00142-2

[16] Burton, H. "Reviews of the progress of dairy science." J. Dairy Res. 35 (1968): 317-330. https://doi.org/10.1017/S0022029900019038

[17] Bell, R. W., and C. F. Sanders. "Prevention of milestone formation in a high-temperature-short-time heater by preheating milk, skim milk and whey." Journal of Dairy Science 27, no. 6 (1944): 499-504. https://doi.org/10.3168/ids.S0022-0302(44)92626-3

[18] Yoon, Jungro, and Daryl B. Lund. "Magnetic treatment of milk and surface treatment of plate heat exchangers: effects on milk fouling." Journal of Food Science 59, no. 5 (1994): 964-969. https://doi.org/10.1111/j.13652621.1994.tb08168.x

[19] Flint, Steve, and Nikki Hartley. "A modified selective medium for the detection of Pseudomonas species that cause spoilage of milk and dairy products." International Dairy Journal 6, no. 2 (1996): 223-230. https://doi.org/10.1016/0958-6946(95)00007-0

[20] Bhutta, Muhammad Mahmood Aslam, Nasir Hayat, Muhammad Hassan Bashir, Ahmer Rais Khan, Kanwar Naveed Ahmad, and Sarfaraz Khan. "CFD applications in various heat exchangers design: A review." Applied Thermal Engineering 32 (2012): 1-12. https://doi.org/10.1016/i.applthermaleng.2011.09.001

[21] Choi, W., S. Jun, L. T. Nguyen, N. Rungraeng, H. Yi, S. Balasubramanian, V. M. Puri, and J. Lee. "3-D milk fouling modeling of plate heat exchangers with different surface finishes using computational fluid dynamics codes." Journal of Food Process Engineering 36, no. 4 (2013): 439-449. https://doi.org/10.1111/j.1745-4530.2012.00684.x

[22] Jun, Soojin, and Virendra M. Puri. "3D milk fouling model of plate heat exchangers using computational fluid dynamics." In 2005 ASAE Annual Meeting, p. 1. American Society of Agricultural and Biological Engineers, 2005. https://doi.org/10.13031/2013.19600

[23] Sharma, Abhishek, and Sandro Macchietto. "Fouling and Cleaning of Plate Heat Exchangers for Milk Pasteurisation: A Moving Boundary Model." In Computer Aided Chemical Engineering, vol. 46, pp. 1483-1488. Elsevier, 2019. https://doi.org/10.1016/B978-0-12-818634-3.50248-4

[24] Stogiannis, I. A., S. V. Paras, O. P. Arsenyeva, and P. O. Kapustenko. "CFD modelling of hydrodynamics and heat transfer in channels of a PHE." Chemical Engineering Transactions 35 (2013): 1285-1290.

[25] Zaza, Afaf, Nour Eddine Laadel, Youness El Hammami, and Mourad Taha Janan. "Numerical study of the fouling effect on wet cooling towers designed to CSP plants." Energy Procedia 157 (2019): 1230-1240. https://doi.org/10.1016/j.egypro.2018.11.289

[26] Luan, H. B., J. P. Kuang, Z. Cao, Z. Wu, W. Q. Tao, and B. Sundén. "CFD analysis of two types of welded plate heat exchangers." Numerical Heat Transfer, Part A: Applications 71, no. 3 (2017): $250-269$. https://doi.org/10.1080/10407782.2016.1264761

[27] Pan, Fei, Xiao Dong Chen, Ruben Mercadé-Prieto, and Jie Xiao. "Numerical simulation of milk fouling: Taking fouling layer domain and localized surface reaction kinetics into account." Chemical Engineering Science 197 (2019): $306-$ 316. https://doi.org/10.1016/j.ces.2018.12.021

[28] Boukhadia, Karima, Houari Ameur, Djamel Sahel, and Mohamed Bozit. "Effect of the perforation design on the fluid flow and heat transfer characteristics of a plate fin heat exchanger." International Journal of Thermal Sciences 126 (2018): 172-180. https://doi.org/10.1016/j.ijthermalsci.2017.12.025

[29] Ameur, Houari. "Effect of the baffle inclination on the flow and thermal fields in channel heat exchangers." Results in Engineering 3 (2019): 100021. https://doi.org/10.1016/i.rineng.2019.100021

[30] Ameur, Houari. "Effect of corrugated baffles on the flow and thermal fields in a channel heat exchanger." Journal of Applied and Computational Mechanics 6, no. 2 (2020): 209-218. 
[31] Georgiadis, Michael C., and Sandro Macchietto. "Dynamic modelling and simulation of plate heat exchangers under milk fouling." Chemical Engineering Science 55, no. 9 (2000): 1605-1619. https://doi.org/10.1016/S00092509(99)00429-7

[32] Pinson, F., O. Gregoire, Michel Quintard, Marc Prat, and Olivier Simonin. "Modeling of turbulent heat transfer and thermal dispersion for flows in flat plate heat exchangers." International Journal of Heat and Mass Transfer 50, no. 7-8 (2007): 1500-1515. https://doi.org/10.1016/j.ijheatmasstransfer.2006.08.033

[33] Sadouk, Hamza Chérif. "Modélisation de l'encrassement en régime turbulent dans un échangeur de chaleur à plaques avec un revêtement fibreux sur les parois." PhD diss., Université Paris-Est, 2009.

[34] Mahdi, Youcef, Abdelkader Mouheb, and Lounes Oufer. "A dynamic model for milk fouling in a plate heat exchanger." Applied Mathematical Modelling 33, no. $2 \quad$ (2009): 648-662. https://doi.org/10.1016/i.apm.2007.11.030 